\title{
Unrestored Electroweak Symmetry
}

\author{
Patrick Meade \\ C. N. Yang Institute for Theoretical Physics, Stony Brook University, Stony Brook, New York 11794, USA \\ Harikrishnan Ramani \\ Berkeley Center for Theoretical Physics, Department of Physics, University of California, Berkeley, California 94720, USA \\ and Theoretical Physics Group, Lawrence Berkeley National Laboratory, Berkeley, California 94720, USA
}

(Received 30 July 2018; revised manuscript received 21 December 2018; published 1 February 2019)

\begin{abstract}
The commonly assumed cosmological history of our Universe is that at early times and high temperatures the Universe went through an electroweak phase transition (EWPT). Assuming an EWPT, and depending on its strength, there are many implications for baryogenesis, gravitational waves, and the evolution of the Universe in general. However, it is not true that all spontaneously broken symmetries at zero temperature are restored at high temperature. In particular the idea of "inverse symmetry breaking" has long been established in scalar theories with evidence from both perturbative and lattice calculations. In this Letter we demonstrate that with a simple extension of the standard model it is possible that the EW symmetry was always broken or only temporarily passed through a symmetry-restored phase. These novel phase histories have many cosmological and collider implications that we discuss. The model presented here serves as a useful benchmark comparison for future attempts to discern the phase of our Universe at $T \gtrsim$ a few $\mathrm{GeV}$.
\end{abstract}

DOI: 10.1103/PhysRevLett.122.041802

Introduction.-Since the discovery of a Standard Model (SM)-like Higgs boson at the LHC [1,2], there have been numerous attempts to understand its implications for physics beyond the SM (BSM) and the cosmological history of the Universe. In particular, the nature of the electroweak phase transition (EWPT) has been investigated in detail due to its possible connection to EW baryogenesis [3]. However, the EWPT is an interesting question to study in its own right. In the SM, using even the simplest original techniques $[4,5]$, there is an EWPT to an unbroken phase at high temperatures. Nevertheless, the potential for the Higgs has yet to have been measured precisely enough to determine whether or not there are differences from the SM prediction of a cross-over. This has driven much work over the past decades, and experimentally determining the shape of the Higgs potential is a compelling driver for future experimental physics programs [6,7]. In particular, testing at future colliders whether the phase transition is consistent with being first-order (FO) is a way to probe whether EW barogenesis is even possible [8]. Additionally, if there was a FOEWPT it would necessarily imply new particles that couple to the Higgs which could be discovered

Published by the American Physical Society under the terms of the Creative Commons Attribution 4.0 International license. Further distribution of this work must maintain attribution to the author(s) and the published article's title, journal citation, and DOI. Funded by SCOAP ${ }^{3}$. by future colliders. There are also cosmic connections for a FOEWPT, as it would create a potentially measurable gravitational wave (GW) signal (see e.g., [9] and references therein). This is especially interesting given that we are now in the era of GW astronomy and the frequency range of interest overlaps the sensitivity of next generation $\mathrm{GW}$ experiments.

While studying the order of the EWPT provides a compelling research program for high energy experimental physics and GW astronomy, there is an even more basic question that can be investigated. Did an EW phase transition ever occur in the early Universe? The original techniques for studying finite-temperature quantum field theory $[4,5]$ gave a robust mechanism for restoring symmetries at high $T$, to the point that it became almost common lore. This stemmed from the fact that scalars acquire a thermal mass at leading order of the form

$$
V(\phi, T) \supset \eta \phi^{2} T^{2},
$$

where $\eta$ represents the coupling of the scalar $\phi$ to other particles or itself. Eventually at high $T$ this term would dominate any negative quadratic combination responsible for spontaneous symmetry breaking and restore the resulting symmetry. However, it was also pointed out in [5] that symmetries need not be restored at high $T$, and there are other restoration or nonrestoration patterns that could be realized in nature, i.e., inverse symmetry breaking (ISB) or symmetry nonrestoration (SNR). While at first these were phenomena 
were discovered perturbatively, they have since been realized on the lattice [10-12] and there is sufficient additional evidence that this possibility is now on firm theoretical footing. Additionally as reviewed in [5] systems exist in nature that demonstrate this phenomenon, such as Rochelle salts and particular liquid crystal systems [13]. Symmetry nonrestoration in generic scalar theories has also been demonstrated in [14-16]. ISB and SNR have also been postulated as solutions to various problems, e.g., the matterantimatter asymmetry with persistent $C P$ violation $[17,18]$, for color-broken phases for baryogenesis in [19], and to avoid monopoles [20-22] and domain walls [23] in grand unified theories (GUTs). Nevertheless, an interesting application that has not been previously successfully demonstrated is applying SNR to the EWS. The fate of EWS with a single Higgs and certain extensions was considered in [24] and symmetry restoration was concluded. Although symmetry nonrestoration was claimed in [25,26] for little Higgs theories, followups in $[27,28]$ have since shown that this occurred through looking at an EFT beyond its validity, and symmetry restoration was concluded at high $T$.

In this Letter we will show that with a simple scalar extension of the SM, a nonrestoration phase can occur for the EWS. The existence of such a phase has a number of implications experimentally and cosmologically. First, the cosmological history is very different than the SM. While it is commonly assumed that all SM particles are massless before the standard EWPT, this is not the case in the phase we describe, and masses increase with temperature. This influences how particles decouple and can provide alternative cosmic histories for relic abundances as well as novel equations of state. Correlated to the temperature-dependent vacuum expectation value (VEV), EW sphalerons are inefficient because of the persistent EWSB and models of baryogenesis which utilize them are not viable. This implies that old mechanisms such as GUT baryogenesis are potentially viable and models of EW baryogenesis are not viable. There also will not be a GW signal, because there is no first-order phase transition. Finally, because the singlets must couple to the Higgs boson, there can be correlated collider signals. However, as we demonstrate, these collider signals can be invisible to future colliders potentially invalidating no-lose theorems for testing the EWPT [8].

Model.-To demonstrate SNR for EWSB we exploit the same term used for symmetry restoration (1). As pointed out in [5], mixed quartics can be negative and this term can cause SNR as well as restoration. In this Letter we consider a SM singlet $s$ transforming in a vector representation of an $O\left(N_{s}\right)$ global symmetry coupling to our Higgs boson through the following Lagrangian:

$$
\mathcal{L}=\mathcal{L}_{\mathrm{SM}}-\frac{\mu_{s}^{2}}{2} s^{2}-\frac{1}{4} \lambda_{s} s^{4}-\frac{\lambda_{h s}}{2} h^{2} s^{2} .
$$

The singlet mass at zero temperature is given by

$$
m_{s}^{2}=\mu_{s}^{2}+\lambda_{h s} v^{2} .
$$

The quartics $\lambda_{s}$ and the SM Higgs quartic $\lambda$ must be positive, but the mixed quartic $\lambda_{h s}$ can be negative. However, to avoid a negative runaway direction, $\lambda_{h s}$, is bounded such that

$$
\lambda_{h s} \geq-\sqrt{\lambda_{s} \lambda} .
$$

Therefore a contribution to (1) for the Higgs boson can be negative from a negative $\lambda_{h s}$ in this range and possibly allow for SNR. Nevertheless, to achieve SNR the negative contribution must outweigh the usual positive contributions. For the Higgs boson there are positive contributions to its thermal mass from SM particles and the Higgs boson itself. At leading order in the high-temperature limit the thermal masses of $h$ and $s$ are given by

$$
\begin{aligned}
\Pi_{h} & =T^{2}\left(\frac{\lambda_{t}^{2}}{4}+\frac{3 g^{2}}{16}+\frac{g^{\prime 2}}{16}+\frac{\lambda}{2}+N_{s} \frac{\lambda_{h s}}{12}\right), \\
\Pi_{s} & =T^{2}\left(\left(N_{s}+2\right) \frac{\lambda_{s}}{12}+\frac{\lambda_{h s}}{3}\right) .
\end{aligned}
$$

The simplest scalar extension, where $N_{s}=1$, requires $\lambda_{h s} \ll-1$ for SNR to overcome the top Yukawa contribution in (5). This requires that $\lambda_{s}$ must be nonperturbative to satisfy (4), which is why this region is usually excluded from interest [8]. However, by taking $N_{s} \gg 1$, it is simple to simultaneously achieve SNR through (5) while maintaining perturbativity of the couplings. In the large- $N_{s}$ limit we can define an effective 't Hooft coupling for the theory

$$
\lambda_{h s}^{c} \equiv\left|\lambda_{h s}\right| N_{s}
$$

which helps organize perturbation theory more clearly. In particular, our condition for SNR will just be that $\lambda_{h s}^{c}$ is greater than some fixed $\mathcal{O}(1)$ value depending on the parameter point. With this coupling definition, our condition for stability of the potential at zero temperature is given by

$$
\lambda_{s} \geq\left(\frac{\lambda_{h s}^{c}}{N_{s}}\right)^{2} \frac{1}{\lambda},
$$

which allows for pertubatively small $\lambda_{s}$ since it only requires that $\lambda_{s} \gtrsim\left(1 / N_{s}^{2}\right)$. In particular, we will use this scaling to define $\lambda_{s}^{c} \equiv \lambda_{s} N_{s}^{2}$, which we will refer to later when further discussing the stability of this phase.

Additionally in this scaling limit, at high $T$ the theory remains only in a partially ordered phase where $\langle s\rangle=0$. This is because the large- $N_{s}$ limit ensures symmetry restoration at high $T$ from (6) regardless of the sign of $\mu_{s}$. (One can easily show that $\Pi_{h}+\Pi_{s}>0$ if (4) is satisfied.) The existence of this phase of the SM has been shown thus far using the leading order expansion in high $T$, and large $N_{s}$. In this regard, the phase is robust as the 


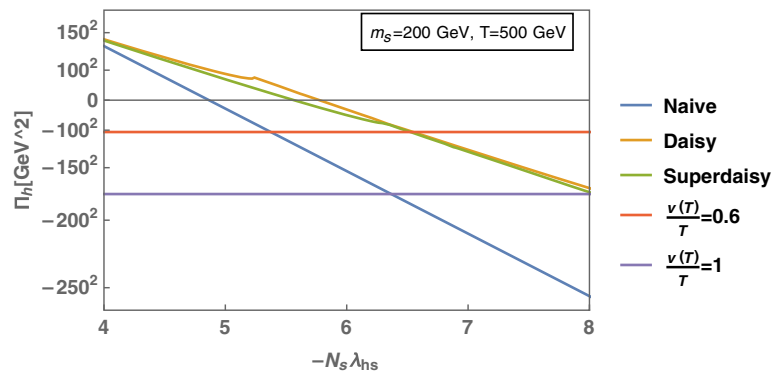

FIG. 1. For an example point with $m_{s}=200 \mathrm{GeV}$ and $N_{s}=600$ we show comparisons between the naive expectations from Eq. (5) and the Daisy and super-Daisy contributions, for $T=500 \mathrm{GeV}$.

couplings can be shown to be as small as required for perturbative control, similar to the arguments made in large $N$ for the $O\left(N_{1}\right) \times O\left(N_{2}\right)$ model in [29]. Note that in the large- $N_{s}$ limit, the expansion parameter which dictates the relevance of super-Daisy diagrams reduces to whether the zero temperature theory is under perturbative control. This theory can be extended to finite $N_{s}$, beyond the Daisy limit, and including finite mass effects using the methods developed in [30] without changing the qualitative conclusions. Additionally, it is interesting to note that some of the typical problematic finite-temperature infrared effects are reduced in a persistent SNR phase since there is never a symmetry restoration (SR) limit which inherently requires massless states near the origin. In Fig. 1 we demonstrate how the effective $\Pi_{h}$ can be quite different than the naive expectation of Eq. (5). In particular, by including these higher-order effects there are many possible thermal histories of our Universe that can be realized. For instance, either $m_{s}$ effects or varying $N_{s} \lambda_{h s}$ can result in the EWS having SNR or other phase histories where one passes from an ordered to disordered and then back to an ordered phase as shown in Fig. 2, which we refer to as a temporary restoration (TR) phase. This can be understood through either the decoupling of thermal effects, or how large of $\Pi_{h}$ results from the coupling constant. It should be emphasized that TR could extend for a very long period if $m_{s}$ is very large. Additionally, more complicated phase histories could exist alternating between SR and NR if additional scalars are appropriately added to the model.

Phase stability.-The existence of SNR, or more complicated phase histories, for the simple model discussed here is robust when looked at from many different vantage points including: RGE stability, thermal decoupling, and thermal fluctuations.

Under RGE evolution for large- $N_{s}$ the 1-loop $\beta$ functions reduce to

$$
\begin{gathered}
\beta_{\lambda}=\beta_{\lambda}^{\mathrm{SM}} \quad \beta_{\lambda_{s}^{c}}=\frac{1}{16 \pi^{2}} 2\left(\lambda_{h s}^{c}\right)^{2}, \\
\beta_{\lambda_{h s}^{c}}=\frac{\lambda_{h s}^{c}}{16 \pi^{2}}\left(12 \lambda+6 y_{t}^{2}-\frac{3}{2} g_{1}^{2}-\frac{9}{2} g_{2}^{2}\right) .
\end{gathered}
$$
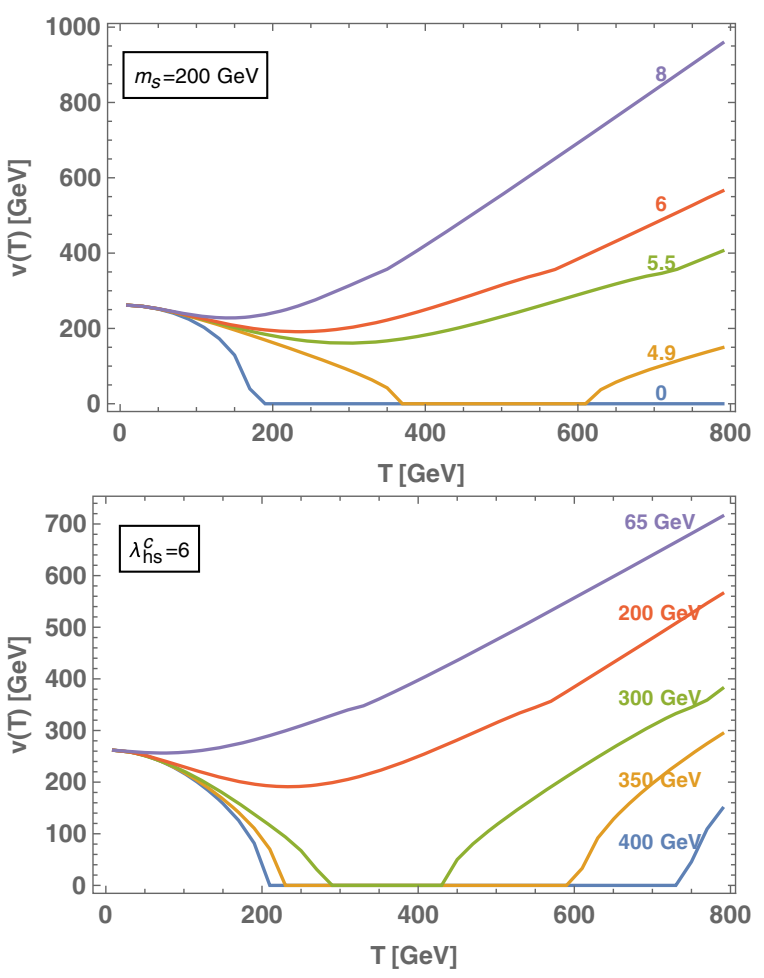

FIG. 2. Top: temperature-dependent VEV, $v(T)$ for different values of $\lambda_{h s}^{c}$ demonstrating different phase histories as a function of the temperature, $T$. Bottom: temperature-dependent VEV for a fixed $\lambda_{h s}^{c}$, the effective coupling, and different values of $m_{s}$. $N_{s}=600$ to exhibit the large- $N_{s}$ limit.

From this basic 1-loop structure it can clearly be seen that the phase structure is robust against RGE evolution. We have numerically verified using 3-loop RGEs that the couplings can remain perturbative to the Planck scale in this model. However, it is important to note that there is still interesting phenomenology that can come out of the solution to the RGE evolution. The effective finitetemperature VEV scales as

$$
v(T) \sim \sqrt{\frac{\mu^{2}-\Pi_{h}(T)}{\lambda(T)}}
$$

from which we can define the ratio

$$
\kappa(T) \equiv v(T) / T
$$

which strongly depends on the value of $\lambda(T)$. In this notation we are defining the thermal $\lambda(T)$ as containing the zero-temperature running coupling constant, as well as the effective change to the quartic from the thermal potential. It is well known that the temperature-dependent contributions to $\lambda$ are small which naively limits the size of $\kappa$ since $\Pi(T) \sim T^{2}$. Nevertheless, at high temperatures the appropriate RGE scale should be $\mu_{R} \sim T$ and the zerotemperature running therefore is important. In particular at 
large- $N_{s}$, the $\beta$-function (9) for $\lambda$ is SM-like and therefore $\lambda(T) \sim 0$ at large $T$ as with the usual metastibility story in the SM. Therefore, large values of $\kappa$ are achievable which can cause a number of interesting phenomenological consequences that we will discuss later. However, there are self-consistency limits on the phase which bound $\kappa$ from above. Despite the SM RGE argument, $\kappa \sim \mathcal{O}(1)$ can be maintained to arbitrary high scales from the $1 / N_{s}$ effects in the $\beta$ function for $\lambda$ while all other couplings remain under perturbative control as well. In all plots in this letter we have chosen $\lambda(T)=\lambda(0)$ for simplicity, but a more dedicated study of the parameter space with all RGE effects for a dynamical scale would be an interesting direction to pursue.

If this phase is robust, then we must also check that the fields stay in thermal equilibrium validating the ansatz of equlibrium local thermal field theory used in describing the phase in the previous section. For our purposes here, it is sufficient that $S$ and $h$ maintain equilibrium since these are the fields which drive the SNR phase. If SM fields were included this would only serve to increase the allowed parameter space for SNR. To establish the validity of thermal equilibrium we can compare the various reaction rates $\quad \Gamma_{h}:(h+h \leftrightarrow h+h), \quad \Gamma_{h s}:(h+h \leftrightarrow s+s)$, $\Gamma_{s}:(s+s \leftrightarrow s+s)$, and permutations thereof to Hubble scale $H$. In the large- $T$ and large- $N_{s}$ limits, the masses of the particles (using only the leading-order contributions to the appropriate П) scale as

$$
m_{h} \sim\left(\frac{\lambda_{h s}^{c}}{12}\right)^{1 / 2} T \text { and } m_{s} \sim \mu_{s}
$$

Therefore at large- $T$ all scalars can be treated as relativistic. The various mixed quartics contribute to $\Gamma_{h}, \Gamma_{h s}$, and $\Gamma_{s}$, but there are also trilinear interactions from the Higgs VEV that matter given the scaling of $v(T)$ for SNR. The trilinear couplings in the large- $T$ limit scales as

$$
g_{h h h} \sim \lambda \kappa T \quad \text { and } \quad g_{h s s} \sim \frac{\lambda_{h s}^{c}}{N_{s}} \kappa T .
$$

For $\Gamma_{h s}$ which provides the strongest constraint, the equilibrium condition is

$$
T \lesssim\left(\frac{\lambda^{2}\left(\lambda_{h s}^{c}\right)^{2} \kappa^{4}}{N_{s}^{5 / 2}}\right) M_{p l}
$$

One can not formally take the infinite- $N_{s}$ limit, since inequilibrium means SNR would not exist in the first place. Note that, in this limit, even a simple scalar $\phi^{4}$ interaction would not stay in equilibrium with itself because of the infinite $N_{s}$ contribution to $H$. Qualitatively taking the $N_{s} \sim \mathcal{O}(100)$ is more than sufficient to maintain perturbativity in the finite-temperature quantum field theory calculations for $T<T_{\mathrm{GUT}}$ depending on the parameter choices, which is the standard decoupling temperature limit for weakly interacting relativistic particles anyway. If $N_{s}$ is lowered even further the range of temperatures where equilibrium holds is only enlarged.

Once the temperature of the Universe drops to the point where $h$ and $s$ are nonrelativistic then they decouple in the usual fashion. Therefore for $T$ between $\max \left(m_{s}, m_{h}\right) \lesssim$ $T \lesssim T_{\mathrm{GUT}}$ thermal equilibrium can be maintained, with the upper limit being reduced for extremely large $N_{s}$. The lower limit unfortunately cannot be reduced further given the parameters of the Higgs sector at zero temperature.

Another concern is the possibility that even though the VEV of the Higgs scales as the temperature $T$, the inherent scale of thermal fluctuations is also of order $T$, so do thermal fluctuations take us out of this new SNR vacuum? Normally this question is asked in the context of a phase transition [31], where the correlation length $\xi$ is used to compare the difference in free energy density, $\Delta f$ between the broken and unbroken vacuums. If the free energy $\Delta F \gg T$ then fluctuations back to the unbroken vacuum become highly improbable. In our case

$$
\Delta F \sim \xi^{3} \Delta f \sim \frac{\left(\lambda_{h s}^{c}\right)^{1 / 2}}{\lambda} T \gg T
$$

so the SNR vacuum is the preferred vacuum that the Higgs boson stays in.

Cosmology.-There are a number of cosmological differences for SNR or TR phases compared to the usual SR phase. Some of these effects are due to the VEV of the Higgs boson not vanishing, while others are more connected to the $\kappa$ parameter space.

1. Gravitational waves: One simple cosmological consequence of SNR is that in the absence of a phase transition there will be no GW signal. However, there will be a difference in how this arises compared to the usual SM statement of a second order phase transition. In our model, there can be shifts to the Higgs couplings relative to the SM and measurable at colliders (discussed in the next section) that could naively imply a GW signal and therefore future GW observatories would be useful to distinguish the phase of the early Universe [32]. In the more complicated TR history there are two phase transitions; however, there are no GW because the phase transitions are second order.

2. Electroweak sphalerons and baryogenesis: EW sphalerons are often a key ingredient in models of baryogenesis ranging from models of EW baryogenesis to leptogenesis. This is due to the fact that they provide a $B+L$ violating process, and are useful as a SM source of baryon number violation or to reprocess a lepton asymmetry into a baryon asymmetry. However, they critically rely on the fact that the EWS is restored at higher temperatures since they are exponentially suppressed by $\sim e^{-4 \pi / \alpha_{w}}$ at zero temperature. However, in a model with SNR or TR, the effective suppression is modified compared to the usual symmetry restoration because 


$$
\Gamma_{\mathrm{sph}} \sim d_{1}\left(\alpha_{w} M_{W}(T)\right)^{4} \exp \left(-\frac{d_{2} \kappa}{\alpha_{w}}\right)
$$

with constants $d_{1} \lesssim \mathcal{O}(1)$ and $d_{2} \gtrsim \mathcal{O}(1)$, implying that in SNR or TR with $\kappa \gtrsim \mathcal{O}(1)$, the exponential suppression persists at high $T$. Only in models of TR would the sphalerons be temporarily active, and then it is a question of model parameters as to whether or not there is sufficient time to generate and/or process a successful baryon asymmetry. There are of course a variety of models that do not require or are unaffected by EW sphalerons, e.g., Affleck-Dine baryogenesis [33] for a field with net $B-L$ and appropriate decay. On the other hand, EW sphalerons strongly constrain models which do not generate a net $B-L$, e.g., $S U(5)$ GUTs, since they wash out $B+L$ generated by out of equilibrium decay of GUT particles. SNR or TR would allow for such a model and for direct $B$ violation at high scales to be a possible baryogenesis explanation. In particular this could allow for compatibility with existing nucleon decay and oscillation experiments which is an interesting direction to pursue theoretically and experimentally. Additionally, since sphalerons in a SR phase are out of equilibrium for $T>10^{12} \mathrm{GeV}$, it is not necessary for SNR or TR phenomena to persist to the GUT scale.

3. Thermal evolution: For the SNR and TR phases, there can be changes to the overall thermal evolution of the Universe through contributions to $H$, novel equations of state for particles which obtain mass through the Higgs boson, and changes in decoupling or recoupling to the thermal bath. For instance, the additional large $N_{s}$ scalars act as radiation and contribute $\rho_{S} \sim N_{s} T^{4}$ to the energy density at early times in addition to the SM radiation bath, and can overwhelm the SM contribution depending on $N_{s}$. However, since temperature-dependent effects do not extend below $T_{\mathrm{EW}}$ it is not necessary for $m_{s}$ to be light in which case the effects can completely disappear well before any measures such as $N_{\text {eff }}$ are relevant. There can also be a contribution to the DM relic density $\Omega_{\mathrm{DM}}$ from $S$. These contributions are a small fraction of the measured $\Omega_{\mathrm{DM}}$, but nevertheless there can be direct detection bounds [8]. The contribution to $\Omega_{\mathrm{DM}}$, and any direct detection bounds, can be turned off through a small $\mathrm{O}(\mathrm{N})$ breaking which allows for decays. This will by definition not alter the SNR and TR phases, but another DM candidate would be needed. One potentially interesting contribution to $H$ from a SNR or TR phase is via the putative vacuum energy contribution from the Higgs potential which is given by $\rho_{V}=-(1 / 48) \lambda_{h s}^{c} \kappa^{2} T^{4}$. This only potentially matters for very large $\kappa$ and as such acts as a self-consistency constraint on the large- $\kappa$ limit. It is also a potentially novel earlyUniverse dark energy given the $T$ dependence. There are no constraints on $\dot{w}_{V}$ at high $T$ and since our contribution does not persist to low $T$ it is not constrained. There could also be additional unknown contributions to the vacuum energy which could modify any conclusion reached about $\rho_{V}$ alone. It would be interesting to study how cosmological perturbations evolve if this contribution were large in the early Universe.

Another interesting cosmological effect concerns the equation of state for any particle which obtains its mass from the Higgs boson. For a particle $i$ with a zerotemperature mass contribution given by $m_{i} \sim c_{i} v$, where $c_{i}$ is the coupling to the Higgs field, at finite temperatures $m_{i}(T) \sim c_{i} \kappa T$. If $\kappa$ is very large the particle is nonrelativistic and does not contribute to $g_{*}$, so for instance the massive EW gauge bosons and top quarks drop out of the SM plasma at early times and alter the evolution of $g_{*}^{\text {SM }}$. There can also be nonstandard evolutions of $w$ as the Universe cools. In typical cosmologies particles either stay relativistic, or change from relativistic to nonrelativistic matter. However, for large $c_{i} \kappa$, the equation of state can change from NR matter at high- $T$ to quasiradiation near $T_{\mathrm{EW}}$ to NR matter at low scales. For moderate $c_{i} \kappa, w$ can asymptote at high- $T$ to a state in between radiation and matter before ultimately acting as NR matter at low temperatures. Depending on the model there could be interesting potential early matter-dominated scenarios for a DM particle that would alter structure formation, or provide a different scaling of $H(a)$.

The SR thermal history is known to keep all particles in thermal equilibrium from around the electroweak scale to $10^{15} \mathrm{GeV}$. All the particles in the SM except for the neutrinos are in thermal equilibrium through contact interactions; as long as the masses are $\mathcal{O}(T)$ or lower, the reaction rates are identical to the SR vanilla cosmology. The Hubble rate, on the other hand, could be different due to the extra degrees of freedom that might be present. As long as $g_{*} \sim N_{s}$ is not astronomically larger than $g_{*}^{\mathrm{SM}}$ (as discussed in the previous section, extremely large $N_{s}$ result in decoupling) we should expect very similar cosmology for $\kappa \sim 1$. However large $\kappa$ could produce novel effects.

Thermal effects can also potentially modify freeze-in and freeze-out calculations which have potential effects on the abundance of both SM and DM particles. Neutrinos are a familiar example via their decoupling caused by the massive $W$ and $Z$ bosons in the SM. In the large- $\kappa$ limit, neutrinos are in equilibrium for

$$
T \leq 10^{-2} \frac{M_{\mathrm{pl}}}{g_{*}^{1 / 2} \kappa^{4}},
$$

unlike in the SM where they were in equilibrium for any $T$ below $\sim 10^{16} \mathrm{GeV}$. For very large $\kappa$ this could lead to neutrinos recoupling at relatively low $T$ (still well above the EW scale) and then decoupling again around an $\mathrm{MeV}$. Unfortunately this will not be measurable in $N_{\text {eff }}$ since even in the extreme $\kappa$ limit the neutrinos rapidly thermalize around $T_{\mathrm{EW}}$ where the SNR/TR effects disappear, but it still 


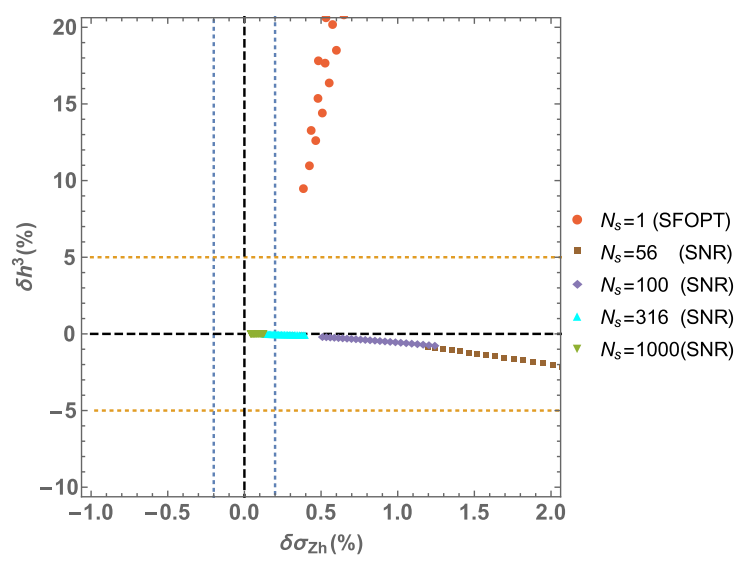

FIG. 3. Comparison between different SNR points and a FOEWPT in the space of $\delta_{Z h}$ and $\delta_{h^{3}}$, for the onset of SNR and $v_{c} / T_{c}=0.6$. Dashed lines represent projected sensitivities that can be found in $[7,8] ; \delta \sigma_{Z h}=0.2 \%$ and $\delta h^{3}=5 \%$.

provides an alternate thermal history for neutrinos. Novel DM scenarios are also possible. DM freeze-out calculations typically have small effects given the scales involved, but this could be altered in a model where the DM mass has a SNR contribution from a sector which is not the Higgs. For freeze-in DM scenarios, bath particles still in equilibrium can be Boltzmann suppressed at temperatures well above their zero-temperature masses, and this could result in a lower freeze-in yield for feebly interacting massive particles (FIMPs), which needs to be compensated by larger couplings for FIMPs to the SM.

Colliders. - The collider phenomenology of this model is very similar to other models with a singlet and a $Z_{2}$ symmetry, such as the nightmare scenario in [8], as it is governed by the singlet-Higgs coupling. For $m_{s} \geq\left(m_{h} / 2\right)$ the interesting collider phenomenology arises in shifts to the $Z$-Higgs coupling $\delta_{Z h}$ and in the triple Higgs coupling $\delta_{h^{3}}$. For lighter $m_{s}$, there is also the possibility of direct production through an off-shell Higgs $\sigma_{h * \rightarrow S S}$. However, the additional multiplicity of scalars $N_{s}$ gives an additional scaling in the collider observables compared to [8]

$\delta_{Z h} \sim N_{s} \lambda_{h s}^{2}, \quad \sigma_{h * \rightarrow S S} \sim N_{s} \lambda_{h s}^{2}, \quad \delta_{h^{3}} \sim N_{s} \lambda_{h s}^{3}$.

When recast in terms of $\lambda_{h s}^{c}$ the observables scale as

$$
\delta_{Z h} \sim \frac{\left(\lambda_{h s}^{c}\right)^{2}}{N_{s}} \sigma_{h * \rightarrow S S} \sim \frac{\left(\lambda_{h s}^{c}\right)^{2}}{N_{s}} \delta_{h^{3}} \sim \frac{-\left(\lambda_{h s}^{c}\right)^{3}}{N_{s}^{2}}
$$

which shows that in the large- $N_{s}$ limit all the observables return to the SM value. The regime $m_{S} \leq\left(m_{h} / 2\right)$ is also potentially viable in the large- $N_{s}$ limit as the contribution to the Higgs partial width

$$
\Gamma_{h \rightarrow s s} \sim \frac{\left(\lambda_{h s}^{c}\right)^{2}}{N_{s}}
$$

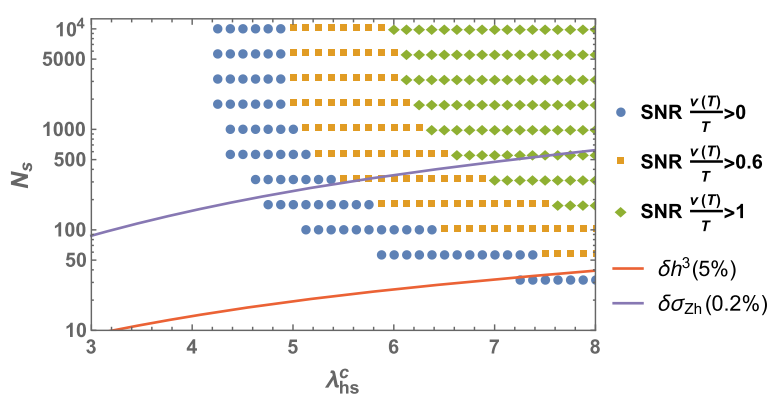

FIG. 4. Collider sensitivities from Fig. 3 in the $N_{s}$ vs $\lambda_{h s}^{c}$ parameter space for $m_{s}=100 \mathrm{GeV}$ superimposed with the SNR parameter space for $T=800 \mathrm{GeV}$ to avoid any finite-mass effects.

scales away. However, it is not particularly interesting from the perspective of SNR or TR since the Higgs boson is already effectively decoupled from the thermal bath for $m_{S}$ in this range.

It is interesting to note that collider measurements consistent with the SM are not sufficient to distinguish between the SM and SNR. Additionally, there can be degeneracies with a FOEWPT as demonstrated in Fig. 3, which may only be disentangled with a future $100 \mathrm{TeV}$ collider measurement of $\delta_{h^{3}}$ or a GW signature. For a given singlet mass, one also can set bounds on $N_{s}$ through precision Higgs measurements as shown in Fig. 4, that can also be correlated with cosmological histories through $\kappa$.

Conclusion.-We have outlined a scenario where EWSB is either persistent in the early Universe or can go through a different order parameter history with respect to temperature. There are a number of interesting potential cosmological consequences that we have outlined for baryogenesis and GW studies, and new cosmological phenomena as well. The cosmological effects in this model are generally small because they are coupled to EWSB which sets a scale in the problem. However, just as SNR and ISB have been used for other applications $[17,18,20-23]$, the cosmological effects could be much larger if ISB and SNR occurred in another sector where the scale and zero-temperature couplings were not predetermined. There are also additional collider signals that we have discussed and that can be correlated with a particular cosmology of interest.

The phases we have shown in this Letter for EWSB are robust in the large- $N_{s}$ limit, but this is also the regime where most collider and cosmological observables can vanish leaving an inherent ambiguity as to the history of our Universe. Therefore it is important to investigate other observables for this model, and also to further study this phase for smaller $N_{s}$. Regardless of the particular part of parameter space, the model for the first time demonstrates successful SNR for EWSB and is an alternative cosmological history consistent with all experimental data. This model will hopefully serve as a benchmark for probing 
early Universe cosmology as we plan for future experiments that look for verifiable information about our Universe for $T \gtrsim$ a few $\mathrm{GeV}$.

We would like to thank David Curtin, Daniel EganaUgrinovic, and Marilena LoVerde for useful discussions. The work of P. M. was supported in part by the National Science Foundation Grant No. PHY-1620628. P. M. would like to thank the Aspen Center for Physics, which is supported by National Science Foundation Grant No. PHY-160761, where part of this work was completed. H. R. is supported in part by the DOE under Contract No. DEAC02-05CH11231.

[1] G. Aad et al., Observation of a new particle in the search for the Standard Model Higgs boson with the ATLAS detector at the LHC, Phys. Lett. B 716, 1 (2012).

[2] S. Chatrchyan et al., Observation of a new boson at a mass of $125 \mathrm{GeV}$ with the CMS experiment at the LHC, Phys. Lett. B 716, 30 (2012).

[3] V. A. Kuzmin, V. A. Rubakov, and M. E. Shaposhnikov, On the anomalous electroweak baryon number nonconservation in the early universe, Phys. Lett. 155B, 36 (1985).

[4] D. A. Kirzhnits and A. D. Linde, Symmetry behavior in gauge theories, Ann. Phys. (N.Y.) 101, 195 (1976).

[5] S. Weinberg, Gauge and global symmetries at high temperature, Phys. Rev. D 9, 3357 (1974).

[6] N. Arkani-Hamed, T. Han, M. Mangano, and L.-T. Wang, Physics opportunities of a $100 \mathrm{TeV}$ proton-proton collider, Phys. Rep. 652, 1 (2016).

[7] R. Contino et al., Physics at a $100 \mathrm{TeV}$ pp collider: Higgs and EW symmetry breaking studies, CERN Yellow Report No. CERN-TH-2016-113.

[8] D. Curtin, P. Meade, and C.-T. Yu, Testing electroweak baryogenesis with future colliders, J. High Energy Phys. 11 (2014) 127.

[9] C. Grojean and G. Servant, Gravitational waves from phase transitions at the electroweak scale and beyond, Phys. Rev. D 75, 043507 (2007).

[10] K. Jansen and M. Laine, Inverse symmetry breaking with 4-D lattice simulations, Phys. Lett. B 435, 166 (1998).

[11] G. Bimonte, D. Iniguez, A. Tarancon, and C. L. Ullod, Inverse symmetry breaking on the lattice: An accurate MC study, Nucl. Phys. B559, 103 (1999).

[12] M. B. Pinto and R. O. Ramos, A nonperturbative study of inverse symmetry breaking at high temperatures, Phys. Rev. D 61, 125016 (2000).

[13] B. Bajc, High temperature symmetry nonrestoration, in Proceedings of the 3rd International Conference on Particle Physics and the Early Universe (COSMO 1999):
Trieste, Italy, 1999 (2000), pp. 247-253 [arXiv:hep-ph/ 0002187].

[14] M. Pietroni, N. Rius, and N. Tetradis, Inverse symmetry breaking and the exact renormalization group, Phys. Lett. B 397, 119 (1997).

[15] M. B. Pinto, R. O. Ramos, and J. E. Parreira, Phase transition patterns in relativistic and nonrelativistic multi-scalarfield models, Phys. Rev. D 71, 123519 (2005).

[16] I. P. Ivanov, Thermal evolution of the ground state of the most general 2HDM, Acta Phys. Pol. B 40, 2789 (2009).

[17] R. N. Mohapatra and G. Senjanovic, Soft $C P$ Violation at High Temperature, Phys. Rev. Lett. 42, 1651 (1979).

[18] R. N. Mohapatra and G. Senjanovic, Broken symmetries at high temperature, Phys. Rev. D 20, 3390 (1979).

[19] M. J. Ramsey-Musolf, G. White, and P. Winslow, Color breaking baryogenesis, Phys. Rev. D 97, 123509 (2018).

[20] G. R. Dvali, A. Melfo, and G. Senjanovic, Is There a Monopole Problem?, Phys. Rev. Lett. 75, 4559 (1995).

[21] P. Salomonson, B. S. Skagerstam, and A. Stern, On the primordial monopole problem in grand unified theories, Phys. Lett. 151B, 243 (1985).

[22] P. Langacker and S.-Y. Pi, Magnetic Monopoles in Grand Unified Theories, Phys. Rev. Lett. 45, 1 (1980).

[23] G. R. Dvali and G. Senjanovic, Is There a Domain Wall Problem?, Phys. Rev. Lett. 74, 5178 (1995).

[24] M. B. Gavela, O. Pene, N. Rius, and S. Vargas-Castrillon, The fading of symmetry nonrestoration at finite temperature, Phys. Rev. D 59, 025008 (1998).

[25] J. R. Espinosa, M. Losada, and A. Riotto, Symmetry nonrestoration at high temperature in little Higgs models, Phys. Rev. D 72, 043520 (2005).

[26] S. Aziz, B. Ghosh, and G. Dey, Broken electroweak phase at high temperature in the littlest Higgs model with T-parity, Phys. Rev. D 79, 075001 (2009).

[27] A. Ahriche, The restoration of the electroweak symmetry at high temperature for little Higgs, 2010, arXiv:1003.5045.

[28] C. Kilic and S. Swaminathan, Can a pseudo-NambuGoldstone Higgs lead to symmetry non-restoration?, J. High Energy Phys. 01 (2016) 002.

[29] J. Orloff, The UV price for symmetry nonrestoration, Phys. Lett. B 403, 309 (1997).

[30] D. Curtin, P. Meade, and H. Ramani, Thermal resummation and phase transitions, Eur. Phys. J. C 78, 7872016.

[31] T. W. B. Kibble, Topology of cosmic domains and strings, J. Phys. A 9, 1387 (1976).

[32] C. Caprini et al., Science with the space-based interferometer eLISA. II: Gravitational waves from cosmological phase transitions, J. Cosmol. Astropart. Phys. 04 (2016) 001.

[33] I. Affleck and M. Dine, A new mechanism for baryogenesis, Nucl. Phys. B249, 361 (1985). 